\title{
Relativistic hydrodynamics in high-energy heavy ion collisions
}

\section{Chiho Nonaka*}

Kobayashi-Maskawa Institute for the Origin of Particles and the Universe (KMI),

Department of Physics, Nagoya University,

Furo-cho, Chikusa-ku, Nagoya 464-8602, Japan

E-mail: nonaka@hken.phys.nagoya-u.ac.jp

Currently a hydrodynamic model is one of promising phenomenological models for description of dynamics of the high-energy heavy ion collisions and a lot of studies based on the hydrodynamic model shows success in understudying experimental data. We develop the new Riemann solver for the Quark-gluon plasma (QGP) fluid. Using the state of the art numerical algorithm for solving the relativistic viscous hydrodynamic equation, we construct a hybrid model of the hydrodynamics and the hadron based event generator, UrQMD. We discuss the QGP property from the investigation of the time evolution of the eccentricities and higher harmonics at RHIC and LHC.

KMI International Symposium 2013 on "Quest for the Origin of Particles and the Universe", 11-13 December, 2013

Nagoya University, Japan

\footnotetext{
* Speaker.
} 


\section{Introduction}

Recently the triangular flow and higher harmonics is one of the hottest topics at Relativistic Heavy Ion Collider (RHIC) and Large Hadron Collider (LHC). The triangular flow and higher harmonics are the coefficients in the Fourier expansion of particle yields as a function of the azimuthal angle,

$$
\frac{d N}{d y d \phi} \propto 1+2 v_{1} \cos \left(\phi-\Theta_{1}\right)+2 v_{2} \cos 2\left(\phi-\Theta_{2}\right)+2 v_{3} \cos 3\left(\phi-\Theta_{3}\right)+2 v_{4} \cos 4\left(\phi-\Theta_{4}\right)+\cdots
$$

Furthermore the results of azimuthal HBT measurements with respect to the second and third order event plane are presented by PHENIX [1]. They extract $\varepsilon_{2}$ and $\varepsilon_{3}$ from the HBT radii which contain information about not only the source shape at freezeout but also the space-time evolution of QGP matter. They show the relation between the initial $\varepsilon_{2,3}$ which are obtained using a Glauber model and the final $\varepsilon_{2,3}$ which are extracted from the HBT radii. They find that the final $\varepsilon_{2}$ from the HBT radii is finite and smaller than the initial $\varepsilon_{2}$. On the other hand, the final $\varepsilon_{3}$ is vanishing, in spite of existence of finite initial $\varepsilon_{3}$. The interesting different response of $\varepsilon_{2}$ and $\varepsilon_{3}$ during space-time evolution gives us a clue to understand the detailed QGP properties.

Currently a hydrodynamic model is one of promising phenomenological models for description of dynamics of the high-energy heavy ion collisions and a lot of studies based on the hydrodynamic model shows success in understudying experimental data (Fig. 1). On the other hand, for the experimental side the variety of the data with high statistics and accuracy are remarkable, which urges us to refine on phenomenological models for analyses of experimental data. In hydrodynamic models, one of the possible improvements is the numerical algorithm for solving the hydrodynamic equation. Unfortunately the numerical algorithm itself has not been paid attention. Here we present the state of the art numerical scheme of causal viscous hydrodynamics for the QGP fluid, which has a shock-wave capturing scheme and less numerical dissipation [2]. Using the hydrodynamic algorithm, we construct a hybrid model of hydrodynamic model plus UrQMD to include the realistic freezeout processes [3].

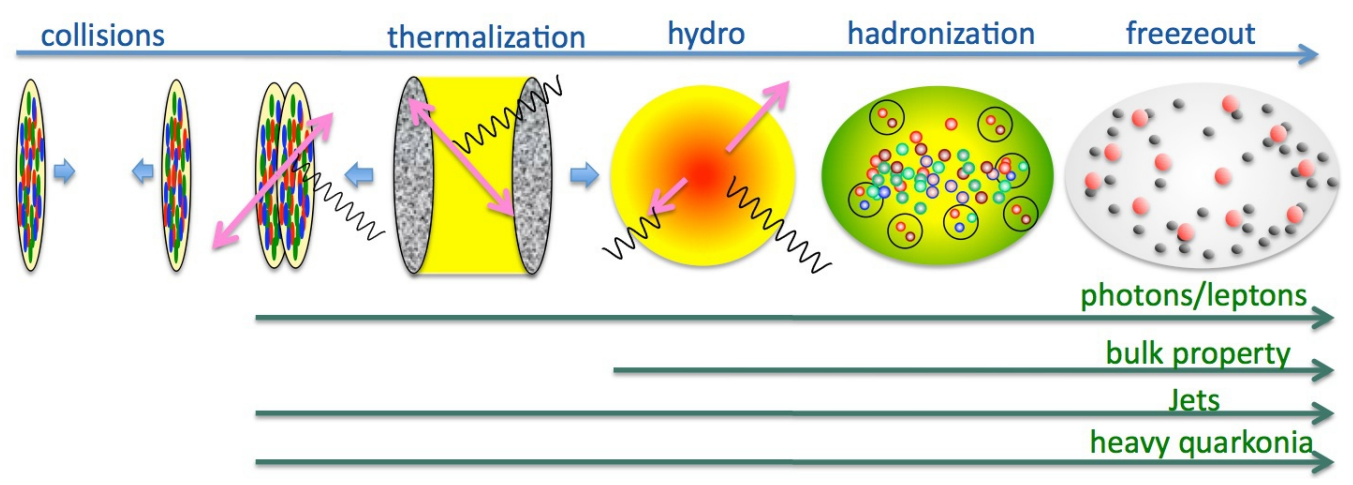

Figure 1: Time evolution of high-energy heavy ion collisions and physical observables at RHIC and LHC. 


\section{Hydrodynamic Model}

The starting point of hydrodynamic models is the relativistic hydrodynamic equation, $\partial_{\mu} T^{\mu v}=$ 0 , where $T^{\mu v}$ is the energy momentum tensor. Currently several relativistic viscous hydrodynamic equations are proposed; Israel-Stewart theory, Ottinger and Grmela, AdS/CFT, Grad's 14momentum expansion, renormalization group and so on. But the problem is that we do not know which equation is suitable for application to the high-energy heavy ion collisions. Here we use the Israel-Stewart theory that is derived based on phenomenological argument. In our algorithm, we decompose the relativistic hydrodynamic equation to two parts, the ideal part and the dissipative part. For solving the ideal part, we propose the new algorithm, a Riemann solver for the QGP fluid based on Godunov method. In addition, we need to solve the relaxation equations coupled with the relativistic viscous hydrodynamic equation. Please see Ref. [2] for the details of our numerical algorithm.

We carry out the several numerical checks and comparison among several algorithms and an analytical solution in shock tube tests, blast wave and so on [2]. Here we show the comparison between the shock tube test of our algorithm and that of SHASTA algorithm that is widely used for application to the heavy ion collisions. In the shock tube test the temperature in the left is 400 $\mathrm{MeV}$ and that in the right is $200 \mathrm{MeV}$ and velocities of both regions are set to zero. Because the analytical solution to the shock tube is known, from the deviation from it the numerical dissipation of an algorithm can be estimated. In the investigation we use the ideal gas equation of state. To evaluate the numerical dissipation, the $L_{1}$ norm which is the sum of the difference between the pressure of numerical calculation and that of the analytical solution is useful. The definition of the $L_{1}$ norm is given by

$$
L\left(p\left(N_{\text {cell }}\right), p(\text { analytic })\right)=\sum_{i=1}^{N_{\text {cell }}} \mid p\left(N_{\text {cell }}\right)-p(\text { analytic }) \mid \frac{\lambda}{N_{\text {cell }}},
$$

where $\lambda$ is the system size and $N_{\text {cell }}$ is the number of cells in the numerical calculation. In the test calculation, we fix the system size $\lambda=10 \mathrm{fm}$. In the left panel of Fig. ?? the number of cell dependence of $L_{1}$ norm for our numerical algorithm and SHASTA is shown. For the each number of cell we find that $L_{1}$ norm of our numerical method is smaller than that of SHASTA, which suggests that our algorithm has the smaller numerical dissipation compared to SHASTA. Next we change the temperature difference between the left and the right in the shock tube test. We find that SHASTA becomes unstable when the temperature in the right becomes $172 \mathrm{MeV}$, though our algorithm is still stable. The SHASTA algorithm has one parameter $A_{\text {ad }}$ with which we can adjust the stability of the numerical calculation and numerical dissipation. The default value of $A_{\text {ad }}$ where the possible maximum value of the numerical dissipation can be subtracted. We change the value from $A_{\mathrm{ad}}=1$ to $A_{\mathrm{ad}}=0.99$, which means that the SHASTA algorithm becomes stable by virtue of the additional numerical dissipation. The comparison between $L_{1}$ norm of our results and that of SHASTA with $A_{\mathrm{ad}}=0.99$ is shown in the right panel of Fig We can see that the difference between them becomes larger than that in the left panel of Fig 2 . The shock tube test calculation suggests that our algorithm has stable even with small numerical dissipation, which is very important and useful property for investigation of high statistics and accuracy experimental data like the higher harmonics. 

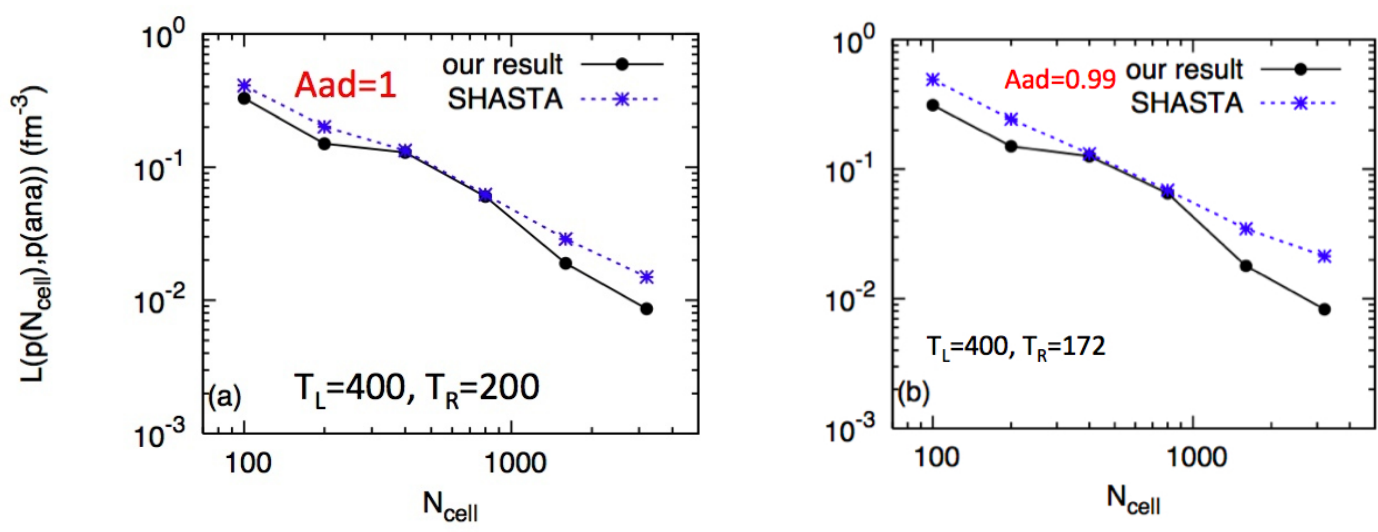

Figure 2: The $L_{1}$ norm of SHASTA with $A_{\mathrm{ad}}=1$ (the left panel) $\left(A_{\mathrm{ad}}=0.99\right.$ (the right panel) ) and our algorithm as a function of number of cell.

\section{Dynamical Model in High-Energy Heavy-Ion Collisions}

Using the state of the art numerical algorithm for solving relativistic viscous hydrodynamic equation, we construct the hybrid model of the hydrodynamic model plus UrQMD. Using the freezeout hyper surface finder [5] and oscar sampler, we prepare the initial condition for the hadron based event generator UrQMD where we can treat the final state interactions appropriately. For initial conditions we use the Monte-Carlo Glauber model (MC-Glauber) and Monte Carlo KLN model (MC-KLN), but here we show the results only from the MC-KLN.

First we investigate the time evolution of higher harmonics in hydrodynamic expansion at RHIC and LHC energies. In the analyses of the higher harmonics we use the following definition for simplicity.

$$
\begin{aligned}
& \varepsilon_{n} e^{i n \Phi_{n}}=\left\langle z^{n}\right\rangle /\left\langle\left|z^{n}\right|\right\rangle \\
& v_{n} e^{i n \Psi_{n}}=\left\langle v^{n}\right\rangle,
\end{aligned}
$$

where $z=x+i y, v=v_{x}+i v_{y}$ and the average is taken with entropy weight. We set the origin so that $\varepsilon_{1}=0$. The definition is different from the actual analyses in experiments, but from time evolution of them the QGP property can be investigated qualitatively.

Here we show the results with MC-KLN initial condition. Please note that these results are obtained with ideal fluid and the Bjorken's scaling solution. The results of MC-Glauber is qualitatively the same as those of MC-KLN, though generally the values of eccentricities of MC-Glauber is smaller than those of MC-KLN. In Fig 3 , the time evolution of $\varepsilon_{n}$ and $v_{n}$ at RHIC is shown. During the whole time evolution the value of $\varepsilon_{1}$ is vanishing because of the definition of the origin. The value of $\varepsilon_{2}$ is larger than those of $\varepsilon_{3}, \varepsilon_{4}$ and $\varepsilon_{5}$. The difference among $\varepsilon_{3}, \varepsilon_{4}$ and $\varepsilon_{5}$ is small. There are minimum point during time evolution of $\varepsilon_{3}$, which suggests that the shape of initial $\varepsilon_{3}$ changes during hydrodynamic evolution. The right panel of Fig. 3 shows the time evolution of $v_{n}$. The growth of $v_{1}$ occurs rapidly and at $t \sim 4.5 \mathrm{fm}$, its value is larger than that of other higher harmonics, which comes from the Bjorken's flow in the longitudinal direction. Other than $v_{1}$, the 
elliptic flow $v_{2}$ is dominant. The values of $v_{n}$ of MC-Glauber are smaller than those of MC-KLN, which suggests that $\varepsilon_{n}$ transforms to $v_{n}$ though hydrodynamic evolution.

In the left panel of Fig. 4 the time evolution of $\varepsilon_{n}$ at LHC is shown. Initial eccentricities at LHC are almost the same as those at RHIC. The life time of the fireball at LHC becomes much longer at RHIC, which makes the detail analyses of changing of $\varepsilon_{n}$ possible. At LHC we find the minimum point in the evolution of $\varepsilon_{3}$, too. In the right panel of Fig. $4 v_{1}$ grows rapidly at the late stage of the time evolution due to the Bjorken's flow. We find that in the beginning $v_{5}$ grows quickly until $t \sim 4.5 \mathrm{fm}$ and suddenly it decreases. On the other hand, $v_{2}$ and $v_{3}$ continue to grow until the end of the time evolutions, which indicates that $v_{5}$ transforms to $v_{2}$ and $v_{3}$. The behavior of $v_{5}$ at LHC is different from that at RHIC.
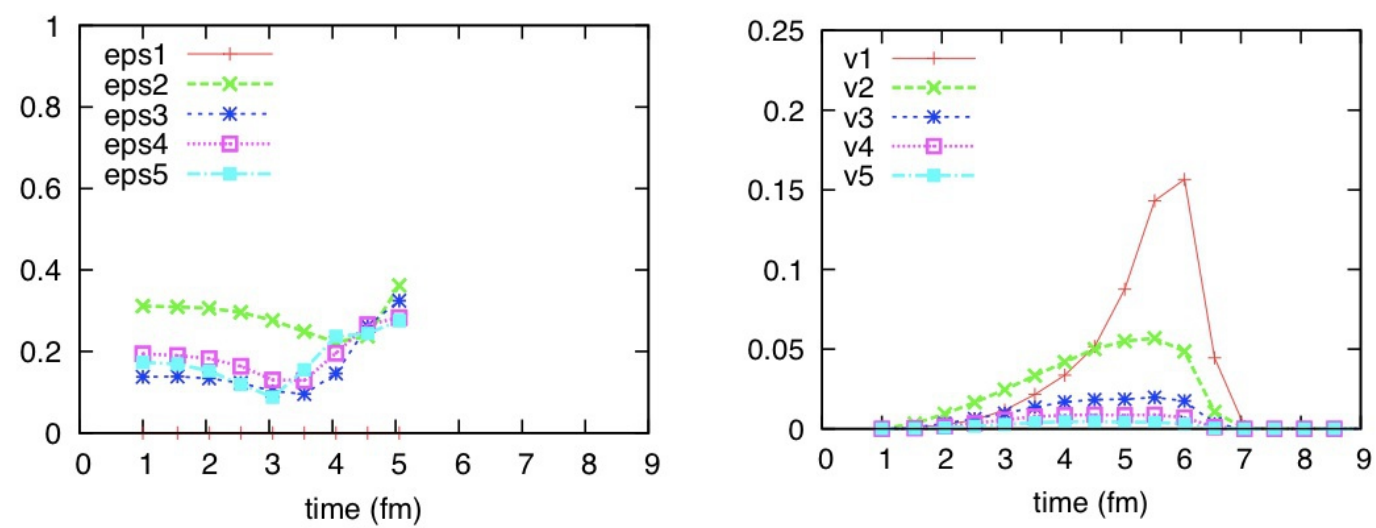

Figure 3: The time evolution of $\varepsilon_{n}$ (the left panel) and $v_{n}$ (the right panel) at RHIC.
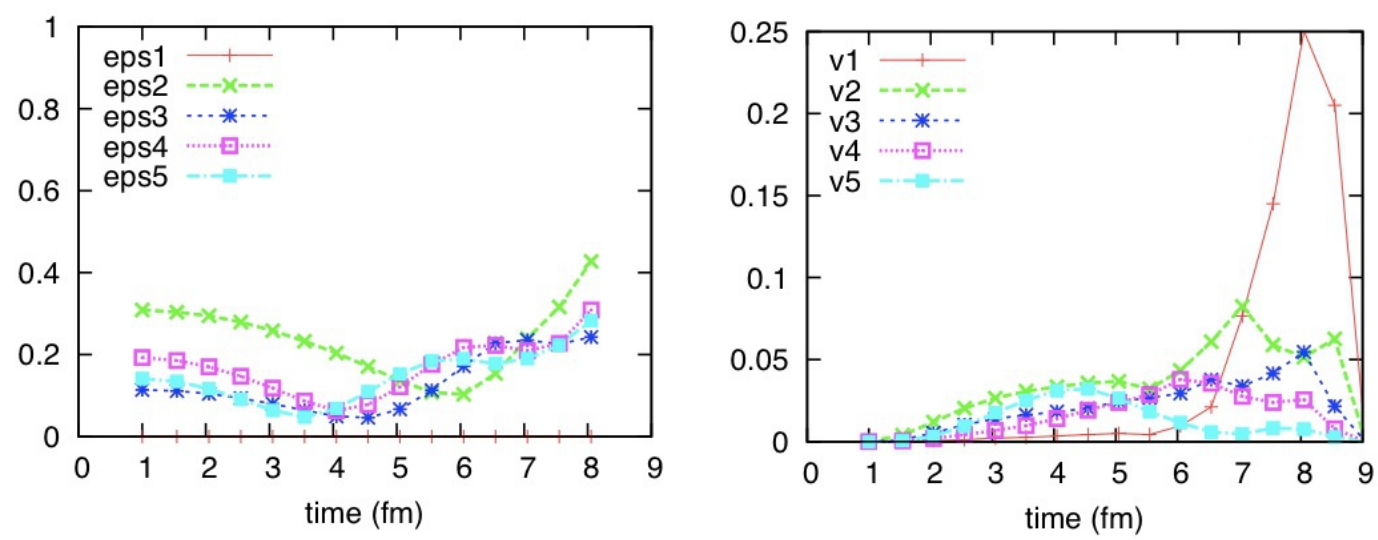

Figure 4: The time evolution of $\varepsilon_{n}$ (the left panel) and $v_{n}$ (the right panel) at LHC.

Finally in Fig. 5 we show the higher harmonics from hydrodynamic evolution and final state interactions at RHIC and LHC. The value of $v_{2}$ at LHC where the fire ball has a longer life time is larger than that at RHIC. On the other hand, $v_{3}, v_{4}$ and $v_{5}$ at LHC are smaller than those at RHIC. This suggests that $v_{n}$ whose origin mainly comes from initial fluctuations smears though the hydrodynamic evolution and final state interactions. 

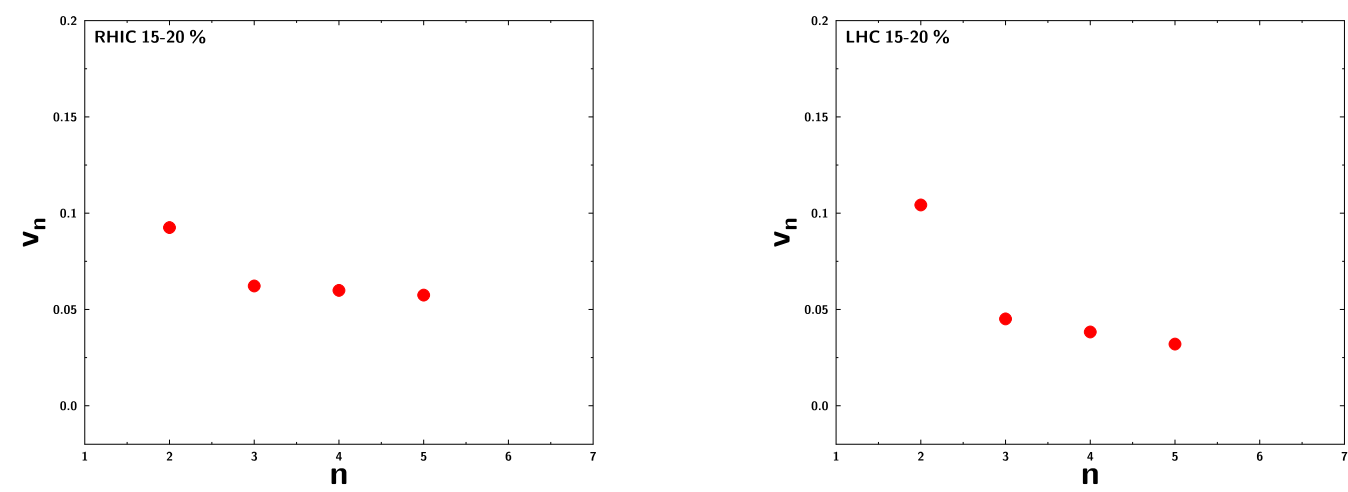

Figure 5: The values of $v_{n}$ from hydrodynamic evolution and final state interactions at RHIC (the left panel) and LHC (the right panel).

\section{Summary}

We constructed the state of the art numerical algorithm for the QGP fluid based on Riemann solver for description of the time evolution of the high-energy heavy ion collisions. We showed that the algorithm is stable even with the small numerical dissipation from the shock tube test, which is very useful and important feature for investigation of experimental data with high statistics and accuracy like the higher harmonics. Using the algorithm, we developed the hybrid model of the hydrodynamic model and the UrQMD. We investigated the time-evolution of the eccentricities and higher harmonics and showed the suggestion of changing sign of $\varepsilon_{3}$ during hydrodynamic evolution, which is found in the experimental data by PHENIX.

\section{References}

[1] T. Niida for the PHENIX collaboration, Nucl. Phys. A 904-905C (2013) pp. 439-442 [arXiv:1304.2876].

[2] Y. Akamatsu, S. Inutsuka, C. Nonaka, M. Takamoto, J. Comp. Phys. (2014), pp. 34-54, [arXiv:1302.1665].

[3] C.Nonaka and S.A.Bass, Phys. Rev. C 75 (2007) 014902 [nucl-th/0607018].

[4] http://www.aiu,ac,jp/ unara/mckln/; T. Hirano and Y.Nara, Nucl. Phys. A743 (2004) 305 (2004) [nucl-th/0404039].

[5] P. Huovinen and H. Petersen, Eur. Phys. J. A 48 (2012) 171 [arXiv:1206.3371 [nucl-th]]. 